\title{
Différenciation Identitaire, Usage et Fonction des Édifices Religieux dans la Gouvernance Urbaine à Meiganga (Nord-Cameroun)
}

\author{
Hamdja Ngoniri Alexis, \\ Lissouck Catherine Laure, \\ Esse Ndjeng, \\ Chercheur INC, Cameroun
}

Doi:10.19044/esj.2020.v16n20p30～URL:http://dx.doi.org/10.19044/esj.2020.v16n20p30

\section{Résumé}

Meiganga est une ville du Nord-Cameroun d'environ 38000 habitants. Elle est caractérisée par une répartition spatiale de l'habitat en deux zones marquées d'une part par des édifices musulmans, et d'autre part, par des édifices chrétiens. Cette évolution de la ville en deux zones distinctes est le résultat de l'absence d'un service public de promotion immobilière. Cela expose la ville à des tensions sociales éventuellement liées à l'accroissement de la valeur économique du sol urbain. Malgré la bipartition religieuse ainsi défini, la ville présente une cohésion sociale apparente. Cela est dû au fait de l'appartenance de la population autochtone à la même et unique communauté traditionnelle Gbaya, dont une partie des ressortissants s'est islamisée et contrôle la chefferie. Toutefois, apparait-il nécessaire que les pouvoirs publics planifient le développement de cette ville en vue de minimiser les tensions qui pourraient naître suite aux velléités expansionnistes de plus en plus prononcées de certaines organisations religieuses. Cette étude est basée sur les données des documents écrits et de diverses enquêtes de terrain. Elle examine tour à tour la politique publique de promotion immobilière au Cameroun, le processus de bipartition de l'espace urbain de Meiganga et l'impact de la disposition des lieux de culte sur la fonctionnalité socio-économique urbaine.

Mots-clés: Politique publique, édifices religieux, gouvernance urbaine, bipartition urbaine, fonctionnalité urbaine 


\title{
Identity Differentiation, Use, and Function of Religious Buildings in Urban Governance in Meiganga (North Cameroon)
}

\author{
Hamdja Ngoniri Alexis, \\ Lissouck Catherine Laure, \\ Esse Ndjeng, \\ Chercheur INC, Cameroun
}

\begin{abstract}
Meiganga is a city in northern Cameroon with a population of around 38,000. Meiganga is characterized by a spatial distribution of housing in two areas marked on the one hand by Muslim buildings, and on the other hand by Christian buildings. The evolution of the city into two distinct zones is due to the absence of a public real estate development service. This exposes the town to social tensions which is eventually linked to the increase in the economic value of urban land. In spite of the religious bipartition thus defined, the city presents an apparent social cohesion. This is due to the fact that the indigenous population belongs to the same one and only traditional Gbaya community, some of whose nationals have Islamized and control the chieftaincy. There appears to be a need for the authorities to plan the development of this city in order to minimize the tensions that could arise from the increasingly expansionist tendencies of certain religious organizations. This study is based on data from written documents and various field surveys. It examines in turn the public policy of real estate development in Cameroon, the process of bipartition of the urban space of Meiganga, and the impact of the layout of places of worship on the urban socio-economic functionality.
\end{abstract}

Keywords: Public policy, religious edifices, urban governance, urban bipartition, urban functionality

\section{Introduction}

D'après les résultats et les projections du Recensement Général de la Population et de l'Habitat (RGPH) rendus publics en avril 2010, le Cameroun compterait à ce jour près de vingt-deux millions d'habitants, avec un taux moyen annuel d'accroissement de 2,8\%. De cette croissance démographique, découle une forte demande en logement sur l'ensemble du territoire national et plus particulièrement en zones urbaines. Face à une pression de moins en 
moins soutenable, le gouvernement a décidé d'accorder une attention particulière au secteur de l'habitat qui s'est traduite par la création des établissements chargés de conduire les projets immobiliers dans les zones urbaines et, dans une moindre mesure, dans les zones rurales. Ainsi, l'implémentation de cette démarche s'est fait suivant quatre périodes.

De 1960 à 1977, c'est la période de l'expérimentation d'une organisation cohérente de l'habitat urbain. Dans plusieurs villes à l'exemple de Ngaoundéré, on procède à des lotissements administratifs (Sabongari, Joli soir...) afin d'accueillir la nombreuse population fascinée par la ville (Esse Ndjeng, 1997). En plus des lotissements administratifs, cette période est marquée par le renforcement des capacités d'investissement de la Société Immobilière du Cameroun (SIC) créée en 1952 par les colons français. Cet engagement des pouvoirs publics à promouvoir l'immobilier se renforce en 1974 avec la promulgation des textes de loi portant régime foncier et domanial. Toutes ces mesures permettent à la SIC de se déployer au point de compte 3 200 logements en 1975 (SIC, 2014). Quoiqu' encourageants, ces résultats s'avèrent mitigés au regard de la forte croissance de la population urbaine nationale que révèle le premier RGPH de 1976, selon lequel, cette catégorie de population est passée de moins de 800000 en 1960 à 1700722 en 1976 (BUCREP, 2010).

De 1977 à 1986, les pouvoirs publics, prenant en compte la pression urbaine toujours plus accentuée, mettent deux établissements sur pied en 1977 à savoir, la Mission d'Aménagement et d'Etude des Terrains Urbains et Ruraux (MAETUR) et le Crédit Foncier du Cameroun (CFC). Pendant que la MAETUR se charge d'aménager, de viabiliser et d'équiper les terrains à bâtir, le $\mathrm{CFC}$ a pour mission de rechercher et de mettre les financements à la disposition des «bâtisseurs » publics et privés. Suite à la création de ces deux structures, l'Etat réorganise la SIC en 1978 pour lui permettre de construire le maximum de logements en saisissant les opportunités offertes par la MAETUR et le CFC. Aussi, fidèle à sa logique de promotion du développement immobilier, l'Etat se dote en 1979 d'un département ministériel principalement consacré à l'encadrement et au suivi des activités des trois établissements susmentionnés. Il s'agit du Ministère de l'Urbanisme et de l'Habitat (MINUH). Le dispositif institutionnel étant en place, le gouvernement se fixe dès fin 1979, l'objectif de construire 10000 logements et d'aménager 30000 parcelles viabilisées sur l'ensemble du pays en trois ans. A l'heure du bilan en 1985, on note un taux de $82 \%$ de logements construits, $100 \%$ de parcelles aménagées et plus de 100 milliards CFA de financements mobilisés.

De 1986 à 2009, le Cameroun connait une conjoncture économique très défavorable au point de rentrer en récession dès 1988. Le Programme d'ajustement structurel dans lequel il s'engage dure pratiquement vingt ans. 
L'insuffisance des financements publics dédiés à la promotion immobilière plombe les activités de tous les acteurs publics du secteur. La MAETUR, le CFC et la SIC résistent à la faillite en changeant de mode de gouvernance. Ils fonctionnent telles des sociétés privées résolument tournées vers la quête du profit. Initialement portées vers la promotion et la production de l'habitat social, ces structures relèvent substantiellement le coût de leurs prestations. Dans les sites d'Olembe et Mfandena à Yaoundé par exemple, les immeubles et autres appartements se vendent à plusieurs dizaines de millions. Cela est un coût largement au-dessus de la bourse du citoyen moyen. L'idée de produire le « logement social » perd de sa substance.

Depuis (2009) et se basant sur les prescriptions du Document Stratégique pour la Croissance et l'Emploi (DSCE), programme d'actions devant conduire le Cameroun à l'" émergence » en 2035, le gouvernement s'est engagé à construire 10000 logements et à mettre à disposition, 50000 parcelles aménagées. En 2016, le bilan de cette opération affiche près de 3000 habitations quasiment terminées à Douala (Mbanga bakoko) et à Yaoundé (Olembé). Aucune parcelle aménagée n'est encore disponible.

En plus de son engagement à bâtir, le gouvernement s'attèle à libéraliser le secteur de l'habitat «social». C'est ainsi qu'environ 50000 logements sont en construction sur l'ensemble du pays par des entreprises chinoises et italiennes notamment. Les initiatives locales sont également motivées par le biais de la Mission de Promotion des Matériaux Locaux (MIPROMALO).

Il convient de reconnaitre que depuis 1960, les bonnes intentions des pouvoirs publics sont perceptibles. Mais, au regard de l'ampleur des besoins, on peut affirmer que l'Etat est encore très loin du « compte ». Car sur les 375 villes (les chefs-lieux d'unités administratives) que compte le Cameroun, à peine une dizaine (les capitales des 10 régions) abrite les investissements de l'Etat et donc la ville de Meiganga ne faisant pas partir. Cette dernière avait déjà anticipé son développement avec le premier chef traditionnel Hamada Boutara (premier à se convertir à l'islam et à porter le titre de Lamido). Durant son règne, il a contribué à la délimitation d'une zone urbaine musulmane, excluant par la même occasion, tous les membres de la communauté Gbaya ayant résisté à l'invasion islamo-peuhle. Quant arrivent les premiers missionnaires chrétiens catholiques et protestants dans les années 1940, ils sont accueillis par les Gbaya non islamisés. Dès lors, la ville de Meiganga se développe en deux parties distinctes, la zone majoritairement peuplée de musulmans à l'Est et la zone majoritairement occupée par les chrétiens à l'Ouest, le centre administratif se situant à l'intersection des deux entités. Cette situation, a entrainé un «laisser-aller» dans le domaine de l'aménagement urbain à Meiganga. L'habitat urbain se trouve ainsi marqué par deux formes d'édifices cultuels liées à des groupes dits chrétiens et 
musulmans. Il découle de ce fait que la ville de Meiganga est à ce jour subdivisée en deux parties, l'une musulmane et l'autre chrétienne. Comment se produit et se perpétue la bipartition à Meiganga ? Quel impact ce phénomène a-t-il sur le développement socio-économique de la ville ?Quelles leçons peut-on tirer de la production urbaine à Meiganga, en rapport avec les atermoiements des pouvoirs publics dans le domaine de la promotion immobilière ?

\section{Méthodologie}

Cette étude s'inscrit dans une approche quantitative. Elle a permis grâce à un questionnaire d'interroger une dizaine d'associations religieuses (soit près de 45 individus) sur le déploiement et les stratégies d'expansion des confessions auxquelles ils appartiennent. En outre, le processus de construction et d'entretien des bâtiments a été abordé au cours d'interview avec 15 techniciens ayant participé à la mise en place des édifices. Cela a permis de préciser les différentes fonctions dévolues ou inhérentes à l'habitat religieux. De plus, les documents consultés ont permis de rassembler les informations sur la création et l'évolution de la ville de Meiganga d'une part et d'autre part, des indications sur le processus d'implantation des différents groupes religieux dans ce milieu. Aussi, avons-nous pu obtenir auprès des services déconcentrés et des structures sous-tutelles du ministère de l'habitat, des rapports traitant de la politique publique en matière de promotion immobilière.

Quant aux données SIG, elles ont été extraites des cartes topographiques publiées en 1956 (disponibles à l'Institut National de Cartographie) et des images satellites de type SRTM (Shuttle Radar Topography Mission) téléchargées en 2016. Les coordonnées GPS de chaque édifice religieux ont été également levées, soit 60 au total. A cela, il faut ajouter des traitements statistiques de type descriptif des données en vue des classifications sur lesquelles les analyses du présent texte reposent. La Figure 1 permet de localiser la ville de Meiganga au Cameroun. 

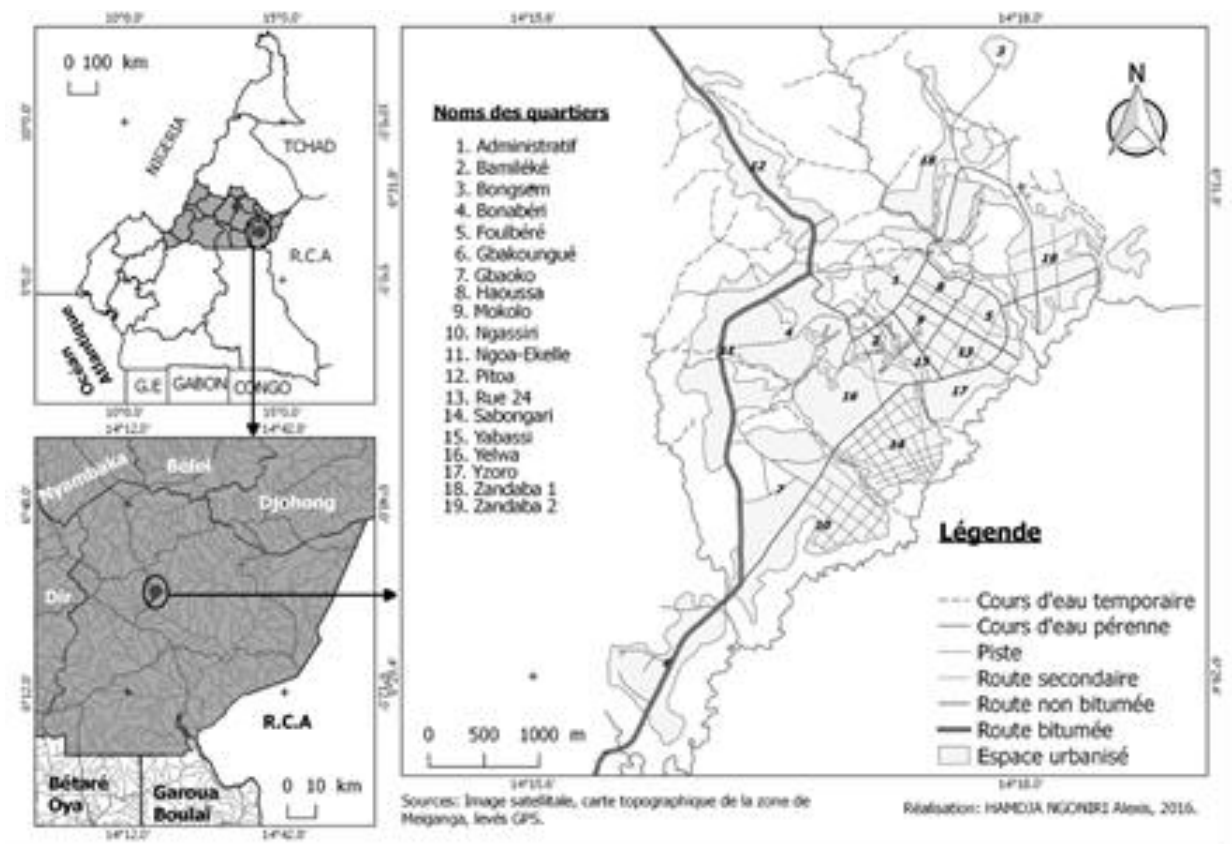

Figure 1. Situation géographique de Meiganga et des quartiers constitutifs de la zone urbaine

\section{Résultats}

1. Bipartition de la zone urbaine de Meiganga : stratégie des croyants

La bipartition de la zone urbaine de Meiganga est caractérisée par deux modes d'occupations. Pendant que les musulmans optent pour un maillage systématique de l'espace, les chrétiens quant à eux occupent l'espace au gré de la multiplication des confessions protestantes (Figure 2). 


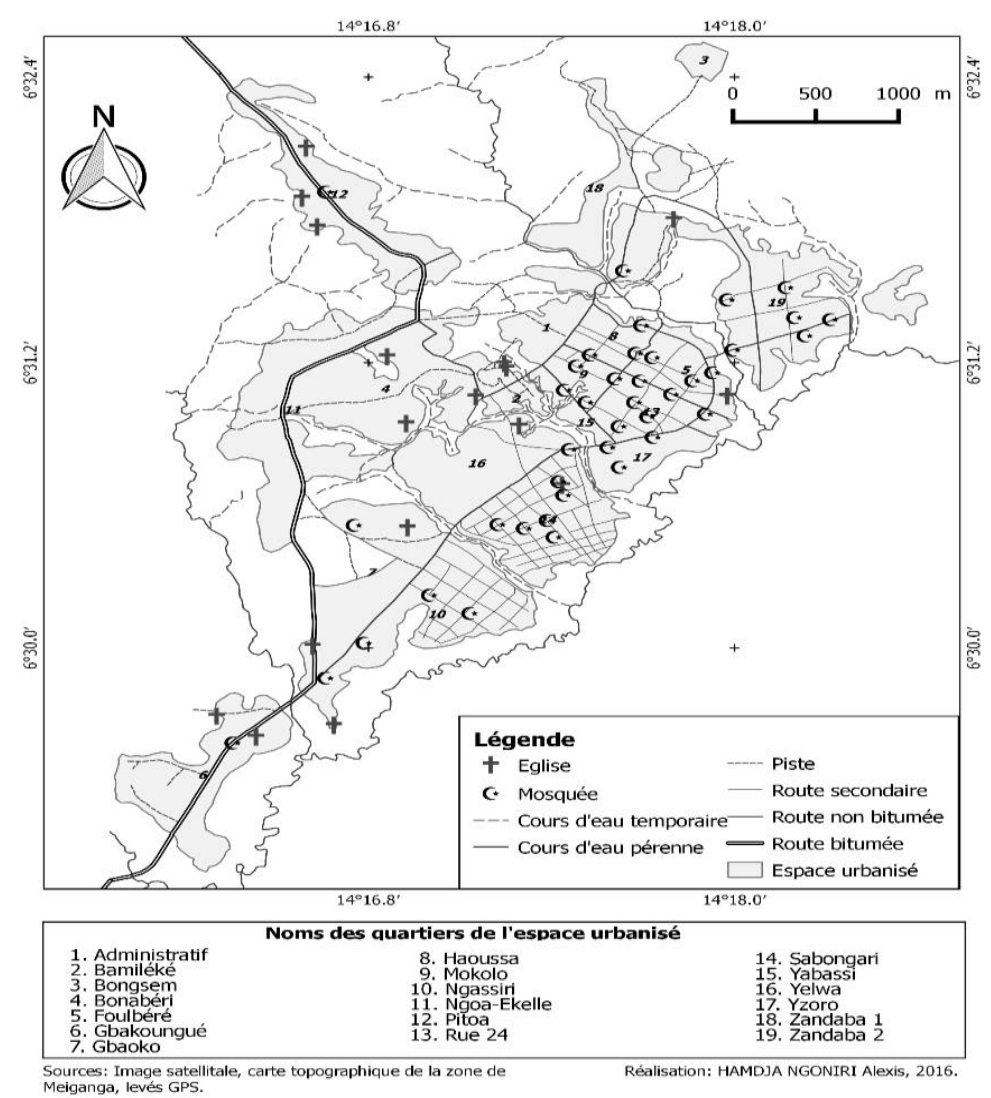

Figure 2. Répartition zonale des mosquées et des Eglises chrétiennes

*S'agissant du maillage systématique de la zone musulmane, il se caractérise par une densification des mosquées de différentes tailles. Elles sont distantes les unes des autres de 150 mètres en moyenne. En fait, chaque groupe familiale (chaque clan) s'arrange à en bâtir une. Outre cette exigence familiale et/ou clanique, tout opérateur économique en quête de reconnaissance et des privilèges sociaux s'arrange à construire une mosquée pour la communauté. De plus, les trois obédiences islamiques (Sunnites, Tidjaniya et Tabligh) tiennent leur autorité politico-spirituelle de leur capacité à se doter des lieux de culte dignes de leurs aspirations de quête de pouvoir et de domination. Dans cette partie de la ville, le Lamido (propriétaire traditionnel des toutes les terres) octroie en priorité des parcelles à des sujets et/ou des groupes de sujets s'investissant dans la multiplication des édifices religieux.

Pour tout dire, la multiplication des mosquées (43 édifices sur à peine 2 kilomètres carrés) et des écoles coraniques participe d'une politique savamment orchestrée par la chefferie traditionnelle en vue de promouvoir l'Islam et à terme, de faire de Meiganga, une ville musulmane.

*Parlant des édifices chrétiens qui se multiplient au gré de l'implantation des confessions protestantes, il faut signaler que dans cette 
zone, il n'existe aucune concertation préalable lors de la mise en place d'un bâtiment. On compte 17 édifices religieux appartiennent à différentes confessions chrétiennes implantées dans la ville. Les principaux groupes sont les catholiques, les adventistes, les messianiques, témoins de Jéhovah et les diverses associations pentecôtistes. Si les catholiques se sont implantés avec l'accord de l'administration coloniale française dans les années 1940, les autres mouvements chrétiens se sont souvent implantés dans les propriétés privées. Ainsi, les fidèles autochtones Gbaya et autres croyants propriétaires mettent des parcelles à disposition. Parmi les 17 propriétés bâties, 11 soit 64 $\%$ relèvent du régime de donations, $4(23 \%)$ de transferts par achat et 2 (13 $\%)$ mises à disposition temporaires.

Parmi les groupes chrétiens, les Témoins de Jéhovah et les pentecôtistes sont particulièrement entreprenants. Ces deux communautés disposent de $50 \%$ des bâtiments religieux dans la ville. Cette présence s'observe également dans la répartition des édifices dans la zone à dominance chrétienne. Ainsi, le challenge du marquage de l'espace urbain est davantage le fait de «nouvelles Eglises».

$\mathrm{Au}$ total, le développement d'une zone urbaine réservée à l'implantation des édifices musulmans est davantage l'initiative de la chefferie et donc du Lamido. Au-delà de ce périmètre, les chrétiens sont libres d'édifier les bâtiments de culte selon leur convenance. Il s'agit donc simplement d'un regroupement spatial des lieux de culte et non, d'une scission sociale. Les paragraphes traitant de la fonctionnalité de la ville y reviennent.

\section{Habitat religieux : de l'usage identitaire aux fonctions urbaines}

L'habitat est l'" organisation des espaces de vie des individus et des groupes », ou encore le «cadre de vie des hommes en société » (Lussault, 2003) $)^{1}$, Dans le présent contexte, l'habitat est perçu comme l'ensemble des édifices permanents dédiés au culte et/ou renseignant sur l'exercice d'une activité à caractère religieux dans un lieu bâti. Ainsi, en plus des édifices de prière, on note l'existence de nombre de bâtiments et de structures connexes (Bernier, 2015) à fonction économique (commerce, élevage...) et sociale (éducation, santé...). Ces aménagements des lieux cultuels (de par les fonctions et/ou l'appartenance religieuse de l'investisseur) leur confèrent des fonctions socio-économiques qui impactent considérablement le développement de la ville. Dans le cas particulier de Meiganga, la mise en place, l'entretien et l'exploitation des immeubles cultuels revêt un caractère économique et social si déterminant qu'il importe de s'y plancher.

${ }^{1}$ Lussault, M. (2003). Dictionnaire de la géographie et de l'espace des sociétés, éd Belin, pp.437- 438 


\subsection{Eléments moteurs de fonctionnalité économique}

La construction et l'aménagement des lieux de culte sont des formes de mise en valeur de l'espace autour desquelles, un circuit économique s'est formé. Plus les projets de construction de bâtiments se mettent en place, plus nombreux sont les métiers et donc les acteurs qui interviennent. En fait, la multiplication des lieux de culte est un vivier d'emploi de BTP (Bâtiment et Travaux Publics) dont l'économie de la ville ne peut se départir. Sur la base des déclarations des Imams et autres chefs de communautés musulmanes, le coût de construction des mosquées varie de 4 millions CFA pour les plus petites d'une capacité de 50 fidèles à 500 millions pour la plus grande qui peut accueillir jusqu'à deux mille croyants (photo 1). Le tableau 1 affiche le coût de construction des mosquées selon la catégorie et la capacité d'accueil.

Tableau 1. Coût de construction des mosquées

\begin{tabular}{|l|l|l|l|l|l|}
\hline $\mathrm{N}^{\circ}$ & Catégorie & $\begin{array}{l}\text { Capacité } \\
\text { d'accueil }\end{array}$ & Nombre & $\begin{array}{l}\text { Coût moyen de } \\
\text { construction (en FCFA) }\end{array}$ & Total (en FCFA) \\
\hline 1 & Petite & $\leq 200$ & 30 & 4000000 & 120000000 \\
\hline 2 & Moyenne & 200 à 500 & 11 & 100000000 & 1100000000 \\
\hline 3 & Grande & $\geq 500$ & 2 & 500000000 & 1000000000 \\
\hline 4 & Total & & 43 & & 2220000000 \\
\hline
\end{tabular}

Source : Enquêtes de terrain, 2016

Les montants ainsi affichés prouvent la détermination des fidèles musulmans à faire prospérer l'Islam dans la ville. Ces lourds financements proviennent à $90 \%$ des contributions locales et à $10 \%$ de l'assistance des confréries saoudiennes.

Quant à la mise en place des édifices cultuels chrétiens, les investigations menées auprès des «Anciens » d'Eglises et du clergé catholique local indiquent un coût global oscillant autour de 200 millions CFA, dont 50 millions pour l'Église catholique. De fait, les chrétiens se montrent plus discrets dès lors qu'on évoque les montants investis dans le cadre de l'aménagement des lieux de culte. Ils affirment tous investir grâce aux contributions des fidèles.

Concernant l'injection de ces fonds dans les circuits économiques, il convient de mentionner que $50 \%$ de ces financements soit plus d'un milliard CFA sont redistribués comme rémunération des techniciens dans les chantiers. Le reste des fonds servant à acquérir le matériel et les matériaux sur place, dans les commerces de la ville.

Après la construction des bâtiments, leur fonctionnement quotidien est assujetti aux travaux d'entretien permanents. Chaque mosquée de taille moyenne mobilise en permanence deux individus rémunérés mensuellement. Ici, le salaire individuel moyen tourne autour de 30000 CFA. Lorsqu'on sait qu'on en dénombre onze en plus des deux grandes mosquées, on comprend aisément qu'une trentaine d'actifs se répartissent environ un million CFA tous 
les mois du fait de l'entretien des mosquées. L'Eglise catholique locale fonctionne sur le même principe au niveau de la paroisse cathédrale.

Pour tout dire, Meiganga n'abritant aucune industrie, et, l'économie de la ville étant basée sur le petit commerce informel, la production de l'habitat religieux est une aubaine pour une nombreuse population active minée par le chômage. Au-delà de l'aménagement des lieux de prière, les confessions religieuses investissent également dans les édifices dédiés à l'éducation et à la santé notamment. Cela contribue à renforcer leur impact dans le développement de l'économie locale.

Photo 1. La mosquée centrale de Meiganga d'une valeur de plus de 500 millions CFA

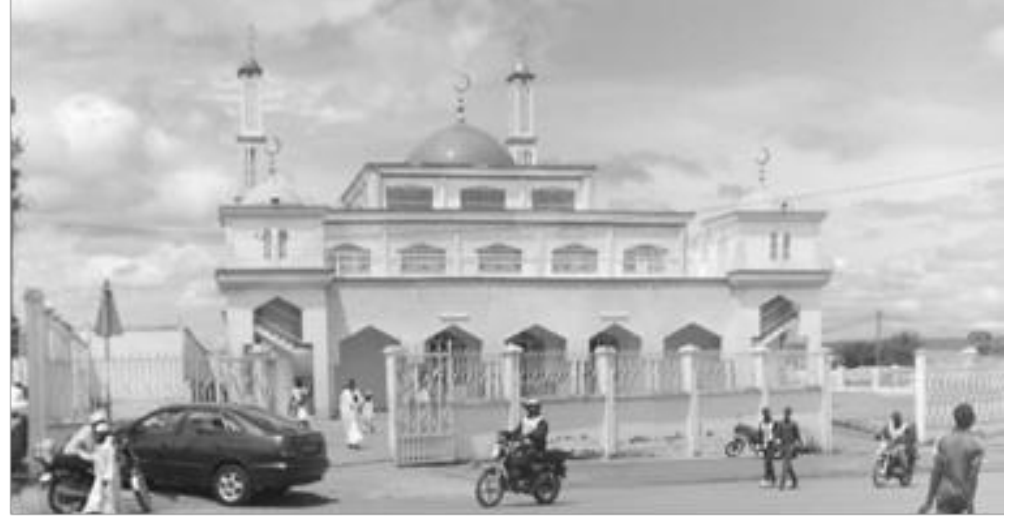

Source : cliché Hamdja, Août 2016

Ce bâtiment date de 2007. Il est situé au lieu-dit Haoussa. C'est un complexe de deux niveaux au dessus du rez-de-chaussée. Il a une capacité d'accueil de 2000 fidèles. Ses parois extérieures de couleur blanchâtre sont repeintes tous les douze mois par des techniciens d'obédiences diverses. Selon l'Imam de service, il a coûté environ 500 millions CFA à la communauté islamique locale. Sa construction a mobilisé plus de 50 individus chrétiens et musulmans pendant 24 mois.

\subsection{Eléments moteurs de fonctionnalité sociale}

Au plan social, la mise en place et l'entretien des édifices religieux sont de grands vecteurs de cohésion urbaine. Certes, les lieux de prière sont réservés aux adeptes de confessions déterminées pendant les heures de culte, mais en ce qui concerne leur mise en place et leur entretien, les experts conviés n'ont point besoin de justifier de la même identité de foi. Selon les dignitaires musulmans interrogés, plus de $55 \%$ d'individus intervenants sur les chantiers «islamiques" sont d'obédience chrétienne. De même, les matériaux de construction nécessaires à l'édification des Eglises sont livrés par des quincaillers musulmans. Donc, du point de vue économique, la multiplication de l'habitat religieux est favorable à tous les habitants de la ville.

Dans le secteur éducatif, les écoles catholique Christ-Roi de NgoaEkelle et protestante pilote de Yelwa sont des modèles de brassage de population. En effet, leur bonne réputation au plan des résultats scolaires en 
fait des lieux de formation privilégiés pour des enfants issus de la bourgeoisie musulmane. Dans l'un et l'autre des cas, les effectifs d'enfants appartenant aux deux obédiences sont pratiquement égaux, avec une tendance de domination musulmane.

En matière de santé et de distribution d'eau, les investissements sont davantage des initiatives de mahométans. La construction du centre de santé intégré privé de Yelwa dans une zone à forte présence chrétienne n'empêche pas qu'on y enregistre une forte domination de patients musulman. Selon les registres de ce centre de santé pour l'année 2015, les malades musulmans (de par les noms) représentent $70 \%$ des individus consultés. Par ailleurs, le service public de distribution d'eau dans la ville étant déficitaire, la communauté musulmane s'est engagée à doter toutes les mosquées de forages (photo 2). Ces points d'eau ravitaillent autant les mahométans que les chrétiens.

En somme, la promotion immobilière par les groupes religieux participe d'une organisation favorable au développement infrastructurel de la ville. Comme l'ont révélé les enquêtes de terrain, ces immeubles étant ouverts et fonctionnels pour toute la population indépendamment des obédiences religieuses, ils concourent à la préservation de la cohésion sociale.

Photo 2. Un forage à proximité d'une mosquée

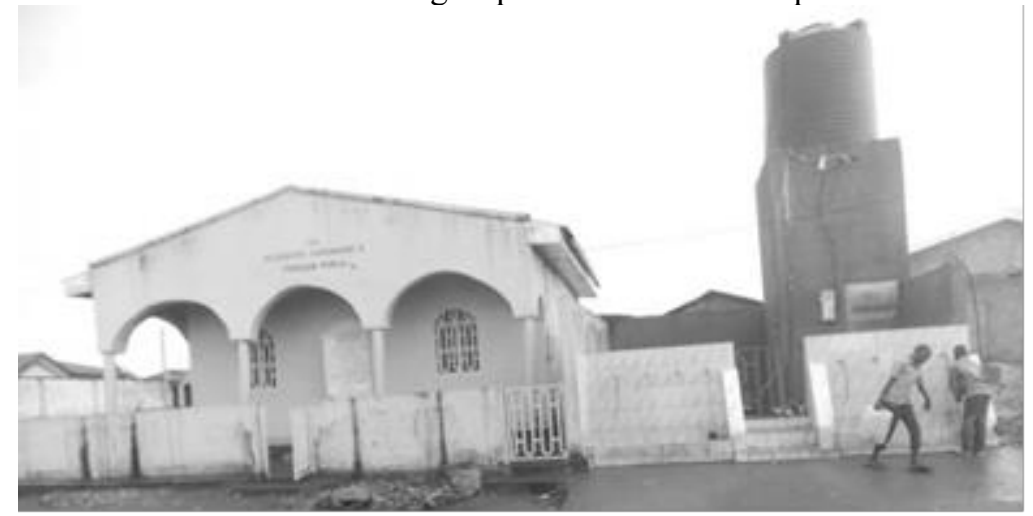

Source: Cliché Hamdja, Août 2016

Ce point d'eau est un prototype de forages aménagés par les fidèles musulmans afin de pallier l'insuffisance d'eau de service public dans la ville. Toutes les populations s'y ravitaillent. Le réservoir en noir au-dessus d'un support en béton, peut contenir 5 mètres cubes d'eau. La communauté islamique de la ville en assure l'entretien. Cette eau est gratuite.

\section{Discussion}

Les résultats de cette étude montrent, la répartition des édifices religieux en deux secteurs à savoir les édifices cultuels musulmans (mosquées) à l'Est de la ville et les édifices cultuels chrétiens (Eglises) à l'Ouest de la ville de Meiganga. Les différentes structures connexes rattachées à ces édifices ont des fonctions socio-économiques (emploi, éduction, santé...) qui contribuent au développement de la ville. Ces résultats vont dans le même sens que ceux 
de Holenstein (2005) selon qui, les organisations religieuses participent de plus en plus au développement local. Elles sont souvent considérées comme des actrices essentielles pour la fourniture de services sociaux et défendent activement les droits des minorités. De ce fait, elles sont invitées à se joindre aux collectivités locales dans leurs efforts de lutte contre la radicalisation et la pauvreté. Pourtant, cette perception des mouvements religieux ne fait pas l'unanimité. Certains auteurs pensent plutôt que la religion entrave l'implication complète des fidèles au processus de développement (Couplet, 2005).

Le présent texte qui traite de la bipartition de l'espace urbain de Meiganga, au regard de la disposition des édifices religieux, en pôles musulman et chrétien corrobore les points de vue de Couplet et Heuchenne (2005) d'une part et de Holenstein, (2005) d'autre part. En effet, la mise en place des immeubles infrastructurels pour pallier aux insuffisances des services publics est effectivement louable parce que cela contribue à l'épanouissement des populations. Mais de manière insidieuse, ces actions procèdent d'une propagande visant le recrutement de nouveaux adeptes. La sympathie suscitée ou recherchée à travers ces investissements attise la compétition voire la concurrence entre les différents groupes d'acteurs. Peu maitrisées, ces attitudes qui frisent l'hypocrisie peuvent générer des tensions sociales. La gouvernance urbaine que définit Le Gales $(1995)^{2}$ comme « un processus de coordination d'acteurs, de groupes sociaux, d'institutions pour atteindre des buts propres discutés et définis collectivement dans des environnements fragmentés, incertains... » peut effectivement pâtir de cette course effrénée vers la multiplication des effectifs de fidèles.

Pour le cas spécifique de Meiganga, les édifices religieux consolident davantage les liens de solidarité entre des populations autochtones du groupe ethnique Gbaya. La mise en place et la valorisation des bâtiments au-delà des considérations religieuses attestent de la force du sentiment d'appartenance à la même entité socio ethnique. D'ailleurs, tous les Gbaya de la ville reconnaissent et se soumettent à l'autorité du Lamido, un Gbaya converti à l'Islam. Alors, par-delà la disposition dans deux zones distinctes des édifices religieux qui obéit plus à la disponibilité foncière qu'à une volonté prononcée de promouvoir une sorte «d'apartheid »dans la localité, cette ville connait une parfaite cohésion sociale.

Seulement, en absence d'un plan d'urbanisme, la ville croît de manière spontanée et rappelle chaque jour davantage l'impératif de planifier son développement. Car, dans le contexte actuel d'expansion de différents types d'extrémismes religieux, il n'est pas exclu qu'un conflit foncier opposant des

${ }^{2}$ Le Gales, P. (1995). Du gouvernement des villes à la gouvernance urbaine, Revue française de science politique, vol. 45, $\mathrm{n}^{\circ} 1$, pp 60 . 
individus ou des groupes d'obédiences différentes en vienne à fragiliser l'équilibre observé. La valeur économique du sol urbain (15 000 FCFA en moyenne par mètre carré) attise des convoitises que seul un parcellaire officiel peut atténuer, épargnant par la même occasion la ville des conflits fonciers interminables. L'intervention de l'Etat dans la promotion immobilière à Meiganga est donc nécessaire pour le maintien de la paix et la cohésion sociale.

\section{Conclusion}

Ce travail a examiné la mise en place des édifices religieux dans deux zones distinctes de la ville de Meiganga. Les différentes analyses faites démontrent que cette implantation des édifices religieux impacte favorablement le fonctionnement de la ville. En effet, sur le plan de la promotion des immeubles infrastructurels, les communautés chrétiennes et islamiques de la ville suppléent effectivement les carences des services publics, sans prétendre devoir les remplacer complètement. La paix et la cohésion sociale fondée sur l'appartenance à la même collectivité tradition Gbaya affermit la croyance en Dieu par-delà la multiplication des confessions. Toutefois, les pouvoirs publics sont vivement attendus sur le terrain de la promotion immobilière et de la planification urbaine, tant la quête du sol dont la valeur économique ne cesse de croître peut fragiliser l'équilibre social actuel.

\section{References :}

1. Bernier, L. (2015). Les Églises et les bâtiments à caractère religieux de Montréal : de la francisation à la patrimonialisation, Thèse de doctorat, Université de grande Bretagne Occidentale, 181p.

2. Bureau Central de Recensement et d'Etude de la population au Cameroun (BUCREP) (2010). « Rapport de présentation des résultats définitifs, Recensement général de la population et de l'habitat du Cameroun en $2005 », 67 p$.

3. Couplet, X. \& Heuchenne, D. (2005). «Religions et bonne gouvernance », $6^{\text {ème }}$ Congrès Européen de Science des Systèmes, du 19 au 22 Septembre 2005, 10p.

4. Esse Ndjeng, M. P. (1997). « Mabanga, un quartier non planifié à la périphérie de Ngaoundéré », Mémoire de Maîtrise de géographie, Université de Ngaoundéré, 86p.

5. Holenstein, A. M. (2005). « Rôle et signification de la religion et de la spiritualité dans la coopération et le développement », Document de réflexion et de travail, $48 \mathrm{p}$. 
6. Le Gales, P. (1995). «Du gouvernement des villes à la gouvernance urbaine », in Revue française de science politique, vol. 45 , n ${ }^{\circ} 1$, pp. 57 95.

7. Lussault, M. (2003). « Dictionnaire de la géographie et de l'espace des sociétés », éd. Belin, 537p, In Trouillard E., 2012, M2 Carthagéo, Rendu dans le cadre du cours de M. Christian Grataloup ,6p.

8. SIC (2014). « Rapport du séminaire d'information et d'évaluation des politiques publiques en matière de promotion des logements sociaux au Cameroun », 16p. 